\title{
Diagnostik erhöhter Leberwerte in der hausärztlichen Versorgung - Eine Befragung zu Voraussetzungen, Vorgehen und erlebten Herausforderungen von Allgemeinmedizinern
}

\section{Evaluation of abnormal liver chemistries in primary care - A survey on the prerequisites, procedure and challenges faced by general practitioners}

\section{(c) (i) $(-)$}

Autoren

Julian Wangler, Michael Jansky

Institut

Zentrum für Allgemeinmedizin und Geriatrie, Universitätsmedizin Mainz

Schlüsselwörter

Leber, Transaminasen, Hausarzt, Primärversorgung, Früherkennung

\section{Keywords}

liver, transaminases, general practitioner, primary care, early detection

eingereicht 18.05.2020

akzeptiert 06.07.2020

online publiziert 24.08 .2020

Bibliografie

Z Gastroenterol 2022; 60: 1203-1211

DOI 10.1055/a-1213-6491

ISSN 0044-2771

(C) 2022. The Author(s).

This is an open access article published by Thieme under the terms of the Creative Commons Attribution-NonDerivative-NonCommercial License, permitting copying and reproduction so long as the original work is given appropriate credit. Contents may not be used for commecial purposes, or adapted, remixed, transformed or built upon. (https://creativecommons.org/licenses/by-nc-nd/4.0/)

Georg Thieme Verlag KG, Rüdigerstraße 14,

70469 Stuttgart, Germany

Korrespondenzadresse

Dr. Julian Wangler

Wissenschaftlicher Mitarbeiter | Zentrum

für Allgemeinmedizin und Geriatrie

Universitätsmedizin Mainz, Am Pulverturm 13, 55131 Mainz, Deutschland

Tel.: ++49/6131/1787 12

Fax: $++49 / 6131 / 176601$

julian.wangler@unimedizin-mainz.de, www.unimedizin-mainz.de/allgemeinmedizin
Univ.-Prof. Dr. med. Michael Jansky

Direktor | Zentrum für Allgemeinmedizin und Geriatrie

Universitätsmedizin Mainz, Am Pulverturm 13, 55131 Mainz,

Deutschland

Tel.: ++49/6131/173240

michael.jansky@unimedizin-mainz.de

\section{ZUSAMMENFASSUNG}

Hintergrund In der hausärztlichen Versorgung treten Leberwerterhöhungen als häufiger Zufallsbefund auf. Neben der Beachtung von Symptomen ist für eine effektive Abklärung entscheidend, welche Leberwerte als Indikatoren einbezogen und wann Patienten zur weiterführenden Diagnostik überwiesen werden. Ebenso kommt es auf eine geregelte Zusammenarbeit zwischen Haus- und Fachärzten an. Bislang fehlt es für den deutschsprachigen Raum an belastbaren Erkenntnissen über das Vorgehen und erlebte Herausforderungen von Hausärzten, wenn es um die Abklärung (unklarer) Leberwerterhöhungen geht.

Methodik Im Zuge einer schriftlichen Befragung, die auf mehreren Vorstudien basiert, wurden zwischen Oktober 2019 und März 2020 insgesamt 2701 Hausärzte in Hessen und BadenWürttemberg zum Vorgehen bei Leberwerterhöhungen befragt. Neben der deskriptiven Analyse erfolgte eine Faktorenanalyse.

Ergebnisse Die Ergebnisse zeigen für die Hausarztmedizin verschiedene Herausforderungen und Problematiken. Hinsichtlich der Beachtung von Warnzeichen sowie mit Blick auf die herangezogenen leberassoziierten Laborparameter sind stark unterschiedliche Cluster hausärztlichen Vorgehens auszumachen. Im Fall erhöhter Leberwerte präferiert eine Mehrheit der Befragten ein kontrolliertes Zuwarten (59\%), allerdings machen Hausärzte im Alltag oft von direkten Überweisungen zu Spezialisten Gebrauch (66\%). In der Zusammenarbeit mit gastroenterologischen Fachärzten bestehen aus hausärztlicher Sicht diverse Schnittstellenprobleme.

Diskussion Es erscheint sinnvoll, Maßnahmen zu ergreifen, die zu einer verstärkten Professionalisierung und Vereinheitlichung der hausärztlichen Diagnostik beitragen und die Zusammenarbeit mit gastroenterologischen Spezialisten besser 
strukturieren. Dazu zählen etwa ein breiteres Angebot von Schulungs- und Fortbildungsformaten, die Entwicklung eines validierten Diagnose- und Behandlungspfads (v. a. Früherkennung von Patienten mit erhöhtem Risiko für eine Leberfibrose oder Leberzirrhose) oder die feste Verankerung einer leberwertassoziierten Blutuntersuchung im Rahmen des Checkups. Der Entwicklung einer fundierten hausarztbasierten Leitlinie zur Erkennung und zum Umgang mit erhöhten Leberwerten sollte nachgegangen werden.

\section{ABSTRACT}

Introduction In primary care, abnormal liver chemistries are often being diagnosed unintentionally. In addition to paying attention to symptoms, it is crucial for an effective clarification which liver values are taken into account as indicators and when patients are referred for further diagnostics. It is also important to have a functioning collaboration between GPs and specialists. To date, there are hardly any current findings about the procedure and the challenges experienced by GPs in German-speaking countries when it comes to clarifying increased liver values.

Methods In the course of a survey based on several preliminary studies, a total of 2,701 GPs in Hesse and Baden-Württemberg were interviewed between October 2019 and March 2020. The focus was on behavior and strategies with regard to the clarification of elevated liver values. In addition to the descriptive analysis, a factor analysis was performed.

Results The results show various challenges and problems that primary care is confronted with in everyday practice. There are very different clusters with regard to paying attention to warning signs as well as liver values that are being analyzed in the course of a liver function test. In the case of increased liver values, $59 \%$ of the physicians surveyed generally prefer a controlled waiting. Nevertheless, many GPs refer patients with elevated liver values directly to gastroenterological specialists $(66 \%)$. The doctors surveyed experience various interface problems in working with gastroenterological specialists.

Discussion It seems sensible to take measures that contribute to greater professionalization and standardization of primary care diagnostics and to a more structured cooperation with gastroenterological specialists. These include, for example, a broader range of training and further education formats, the development of a validated diagnostic pathway for classifying and evaluating elevated liver enzymes (especially early detection of patients at increased risk for liver fibrosis or liver cirrhosis) or the establishment of a liver function screening as part of the general medical check-up. The development of a GP-based guideline for dealing with increased liver values should be followed up.

\section{Einleitung}

In der hausärztlichen Versorgung stellt die Analyse von Leberwerten eine der häufigsten Laboruntersuchungen dar. Dabei ist die Prävalenz für erhöhte Leberwerte unter primärärztlich versorgten Patienten in Deutschland unklar [1]. Im Rahmen der GutenbergHerz-Studie wurden bei jedem fünften Patienten Leberwerterhöhungen festgestellt [2]. Die SHIP-Studie konnte eine ALT-Erhöhung für jeden vierten Patienten nachweisen [3].

Verschiedene Arbeiten konnten belegen, dass Leberwerterhöhungen mit vermehrter Mortalität einhergehen [4-9]. Häufige Ursachen sind Alkoholabusus, Medikamenteneinnahme, die nichtalkoholische Leberverfettung und Virushepatitiden [10-13]. Da erhöhte Leberwerte auf vital bedrohliche Erkrankungen hinweisen können, kommt es auf eine frühzeitige Abklärung und zielgerichtete Therapie an.

In den häufigsten Fällen sind Hausärzte die ersten Behandler, denen (unklar) erhöhte Leberwerte als (Zufalls-)Befund im Rahmen einer Routinekontrolle auffallen [14-16]; zudem obliegt ihnen die Rolle des Zuweisers für weitergehende diagnostische und therapeutische Maßnahmen. In Anbetracht der Zeit- und Ressourcenknappheit in der allgemeinärztlichen Versorgung stellt sich eine differenzialdiagnostische Abklärung zur Früherkennung von Lebererkrankungen für Hausärzte anspruchsvoll dar [17, 18]. Dies betrifft nicht nur die ätiologische Einordnung und Bewertung spezifischer erhöhter Leberwerte, sondern schließt auch die Berücksichtigung anderer möglicher Warnzeichen ein [19]. Neben der Frage, welche Werte in welchen Referenzbereichen und Kon- stellationen als aussagekräftige Indikatoren einbezogen werden $[16,18,20]$, ist für den hausärztlichen Umgang mit (unklar) erhöhten Leberwerten eine Differenzierung ausschlaggebend, in welchem Fall ein abwartendes Offenhalten (mit Wiederholung des Labors) geboten und wann eine sofortige Abklärung indiziert ist, z. B. durch direkte Überweisung zum Facharzt oder zu einer Leberambulanz [16-18].

Nicht nur im deutschsprachigen Raum, sondern auch in anderen europäischen Ländern wird die primärärztliche Versorgung für mangelnde Effektivität bei der konsequenten Identifizierung und Abklärung erhöhter Leberwerte kritisiert [2, 14, 20, 21]. So wird zum einen ein geringer Anteil an Frühdiagnosen moniert, zum anderen ein uneinheitliches und stark vom einzelnen Hausarzt abhängiges differenzialdiagnostisches Vorgehen. Ein Grund dafür wird im Fehlen strukturierter, zielgerichteter Früherkennungsprogramme für chronische Leberkrankheiten im Rahmen der Regelversorgung gesehen [22]. Zudem mangelt es an einem evidenzbasierten und breit etablierten Diagnose- und Behandlungspfad, der Hausärzte bei der Erkennung, Einordnung und Bewertung von Leberwerterhöhungen unterstützt, insbesondere mit Blick auf Patienten mit hohem Risiko für eine Leberzirrhose [10, 18, 23]. Internationale Arbeiten lassen vermuten, dass ein belastbarer, in der Breite der Versorgung angewandter Diagnosealgorithmus entscheidende Vorteile generieren könnte, darunter KostenNutzen-Effekte, eine raschere Früherkennung, eine effektivere Verlaufsbegleitung und individuell passgenauere Therapiemaßnahmen, die ein Fortschreiten der Krankheit verhindern und sogar zu einer Zirrhoseregression führen können [24-28]. Für Deutsch- 
land kommt hinzu, dass Allgemeinmediziner bislang nicht auf explizit hausarztbasierte, evidenzgeprüfte Leitlinien zurückgreifen können.

Bislang liegen für den deutschsprachigen Raum keine systematischen Untersuchungen vor, die die primärärztliche Versorgungsrealität bei Leberwerterhöhungen erfassen. Damit einhergehend fehlen Erkenntnisse zu hausärztlichen Vorgehensweisen im Umgang mit (unklar) erhöhten Leberwerten, zu technisch-apparativen Voraussetzungen und möglichen Schnittstellenproblemen zwischen Haus- und Fachärzten. Ziel der Querschnittsstudie ist es, die Primärversorgung in besagten Zusammenhängen eingehender zu beleuchten, um daraus Schlussfolgerungen für den Status quo und die Optimierung der Leberzirrhose-Diagnostik abzuleiten.

\section{Erkenntnisinteresse}

Im Zentrum der Studie stehen folgende Fragestellungen:

- Welche Voraussetzungen bestehen in der hausärztlichen Versorgung hinsichtlich der Erkennung und Abklärung von Leberwerterhöhungen?

- Wie gehen Hausärzte bei der Einordnung und Abklärung (unklar) erhöhter Leberwerte vor?

- Wie wird die Zusammenarbeit mit fachärztlich-internistischen und gastroenterologischen Fachärzten bei der Behandlung von Patienten mit Leberwerterhöhungen erlebt? Inwiefern bestehen dabei Herausforderungen?

- Welche Maßnahmen zur Verbesserung der Früherkennung von Lebererkrankungen im primärärztlichen Setting werden von Hausärzten befürwortet?

\section{Methodik}

\section{Studiendesign und Setting}

Die vorliegende Studie basiert auf einer Befragung, die im Frühjahr 2017 durchgeführt und konzeptionell erprobt wurde. Besagte Untersuchung fand im Rahmen des vom Gemeinsamen Bundesausschuss geförderten Projekts SEAL (Structured Early Assessment for Asymptomatic Liver Cirrhosis) zur Früherkennung einer Leberfibrose oder asymptomatischen Leberzirrhose statt. Dabei wurden 391 rheinland-pfälzische Allgemeinmediziner zu ihrem Vorgehen bei Leberwerterhöhungen befragt. Dann wurde der Fragebogen aktualisiert und die Studie in deutlich größerem Maßstab neu aufgelegt, um zu eruieren, inwiefern sich die damaligen Resultate bestätigen lassen. Angesichts des stark lückenhaften Forschungsstands hinsichtlich der Versorgung von Lebererkrankungen im niedergelassenen Bereich wurde die Befragung bewusst als explorative Studie angelegt.

\section{Rekrutierung und Teilnehmer}

Die anonymisierte Befragung wurde zwischen Oktober 2019 und März 2020 durchgeführt. Auf schriftlichem Weg zur Teilnahme eingeladen wurden sämtliche als Behandler aktive Hausärzte in Hessen (3.839) und Baden-Württemberg (6.664). Die teilnehmenden Hausärzte erhielten keine Aufwandsentschädigung.

\section{Erhebungsinstrument und soziodemografische Variablen}

Der im Jahr 2017 ursprünglich entwickelte und durch eine Gruppendiskussion mit 10 Hausärzten abgestützte Fragebogen wurde unter Berücksichtigung zweier Vorstudien [34, 35] sowie einer Literaturrecherche aktualisiert und weiterentwickelt. Er orientiert sich in seinen Schwerpunkten an den genannten Forschungsfragen. Die Ausfülldauer beträgt ca. 12 Minuten.

Als soziodemografische Merkmale wurden Alter, Geschlecht, Niederlassungsmodell, Zahl der Ärzte, Patienten pro Quartal und Praxisumgebung erhoben. Die genauen Regionen und Landkreise, in denen die Praxen sich befinden, wurden aus Gründen der Anonymisierung nicht abgefragt und können jenseits der allgemeinen Angabe der Praxisumgebung nicht ausgewiesen werden.

\section{Datenanalyse}

Nach Bereinigung des Datensatzes wurden die Daten mittels SPSS 23.0 ausgewertet. Um unterschiedliche Vorgehensweisen von Hausärzten sichtbar zu machen, wurde auf das Verfahren der Faktorenanalyse (Varimax-Rotation) zurückgegriffen, bei der Variablen aufgrund von systematischen Beziehungen (Korrelationen) untereinander zu Faktoren zusammengefasst werden [29]. Im Vorfeld wurden die Voraussetzungen für die Faktorenanalyse geprüft (Stichprobeneignung nach Kaiser-Meyer-Olkin, signifikantes Ergebnis bei Bartlett-Test auf Sphärizität, Kommunalitäten aller eingeschlossenen Variablen über Grenzwert, 5). Als Grenze, ab der ein Item auf einen Faktor lädt, wurde der Wert $.4 /-.4$ gewählt [29].

\section{Ergebnisse}

\section{Stichprobe}

Von den 2.733 bearbeiteten Fragebogen gingen 2.701 vollständig ausgefüllte Bogen in die Auswertung ein (Gesamtrücklauf: $26 \%{ }^{1}$ ). Die Stichprobe ist wie folgt strukturiert:

- Geschlecht: $61 \%$ männlich, 39\% weiblich

- Durchschnittsalter: 52 (Median: 53, SD: 9,5)

- Praxisumgebung: 49\% mittel- und großstädtisch, 51 \% ländlich-kleinstädtisch

- Praxisform: 51 \% Einzelpraxen, 46\% Gemeinschaftspraxen, $3 \%$ Sonstiges

- Patienten pro Quartal: 19\%<1.000, $38 \%$ 1.000-1.500, $19 \% 1.501-2.000,24 \%>2.000$

\section{Apparative und diagnostische Voraussetzungen}

29 \% der Befragten geben an, in der eigenen Praxis neben der GKV-Früherkennungsuntersuchung eine spezielle Leber-Checkup-Untersuchung zur Früherkennung anzubieten. Zwei Drittel (66\%) halten kein solches Untersuchungsangebot als Zusatz zum GKV-Gesundheits-Check-up vor.

1 Rücklauf nach Bundesland: $27 \%$ (1.040) Hessen, $25 \%$ (1.661) BadenWürttemberg. 
In Bezug auf die vor Ort bestehenden apparativen Voraussetzungen machen 89 \% die Angabe, in der eigenen Praxis über die Möglichkeit einer Oberbauchsonografie zu verfügen. Weitere $64 \%$ können auf eine erweiterte Labordiagnostik zurückgreifen ${ }^{2}$. $5 \%$ bieten eine Untersuchung mittels Elastografie an.

$39 \%$ der Befragten bekunden, sich bei der Abklärung von Leberwerterhöhungen häufig oder gelegentlich an Handlungsempfehlungen medizinischer Fachgesellschaften (Praxisempfehlungen, Expertisen, Leitlinien) zu orientieren (20\% selten, $38 \%$ nie, $3 \%$ weiß nicht).

\section{Symptomatiken und diagnostisches Vorgehen}

Die Befragten schätzen, dass durchschnittlich 19\% der Patienten in ihrer Praxis von Leberwerterhöhungen betroffen sind (Minimum: 14\%, Maximum: $26 \%$ ). Analog zur häufig unspezifischen Symptomatik geben die Befragten aus ihrer Erfahrung an, dass durchschnittlich $41 \%$ der Patienten, bei denen ihnen erhöhte Leberwerte aufgefallen sind, zugleich klinische Symptome hatten.

Im Rahmen einer Itembatterie wurde nach häufigen Hinweisen auf eine beginnende Leberkrankheit gefragt, die üblicherweise zu tiefergehender Diagnostik veranlasst. Aus ihrer bisherigen Erfahrung achten Hausärzte dabei besonders auf einen übermäßigen Konsum von Alkohol, aber auch auf Anzeichen wie Oberbauchbeschwerden, Müdigkeitserscheinungen, Aszites, Juckreiz und Hautveränderungen (vgl. > Tab. 1).

Einer der Schwerpunkte der Befragung liegt auf der hausärztlichen Analyse von Laborwerten, die im Zusammenhang mit Lebererkrankungen stehen können. Es zeigt sich, dass Hausärzte bei einer allgemeinen Screening-Untersuchung v. a. die $\gamma$-GT als Laborwert fokussieren. Rund zwei Drittel beziehen die AspartatAminotransferase (ASAT, AST, GOT) in ihre Untersuchung ein, gefolgt von der Alanin-Aminotransferase (ALAT, ALT, GPT) und der alkalischen Phosphatase (AP), der Thrombozytenzahl sowie dem MCV (vgl. > Tab. 2, Gesamtzustimmung).

Eine Faktorenanalyse nach Varimax-Methode, deren statistische Voraussetzungen erfüllt waren, zeigt, wie heterogen sich das hausärztliche Vorgehen bei der Analyse von Leberwerten darstellt. So betrachtet ein Cluster von Ärzten besonders Funktionsparameter wie Bilirubin, Quick (INR), Cholinesterase und Albumin, wohingegen in einem anderen Cluster Indikatoren für eine Zellschädigung analysiert werden. Neben weiteren Parametern findet hier v. a. die Alanin-Aminotransferase Beachtung. Zudem fällt ein dritter Cluster auf, der auf die $\mathrm{y}$-GT als Parameter für eine mögliche Leberkrankheit fokussiert.

Die Befragten erhielten die Nachfrage, was ihrer Auffassung nach die drei wichtigsten und aussagekräftigsten Indikatoren zur Früherkennung einer Leberzirrhose sind (Mehrfachnennungen waren möglich). Analog zu den bisherigen Ergebnissen werden daraufhin y-GT (92\%), Aspartat-Aminotransferase (83\%) und Alanin-Aminotransferase (79\%) genannt. Andere Werte folgen deutlich dahinter.

2 Bei der erweiterten Laboruntersuchung werden neben dem Blutbild auch Leber- und Nierenwerte sowie Elektrolyte bestimmt. Dieser erweiterte Laborcheck wird als sogennante IGeL-Leistung i. d. R. privat in Rechnung gestellt.
- Tab. 1 Hinweise auf beginnende Lebererkrankungen. Frage: Was sind für Sie die häufigsten Hinweise auf eine beginnende Lebererkrankung und veranlassen Sie zu einer tiefergehenden Diagnostik? $(\mathrm{N}=2.701)$.

\begin{tabular}{|c|c|}
\hline & Gesamtzustimmung \\
\hline Langjähriger Alkoholkonsum & $94 \%$ \\
\hline Vermuteter Alkoholmissbrauch & $78 \%$ \\
\hline Oberbauchbeschwerden & $76 \%$ \\
\hline Müdigkeit, Abgeschlagenheit & $75 \%$ \\
\hline Aszites & $71 \%$ \\
\hline Chronischer Juckreiz & $71 \%$ \\
\hline Leberhautzeichen & $65 \%$ \\
\hline Appetitverlust & $55 \%$ \\
\hline Gewichtsveränderungen & $51 \%$ \\
\hline Multiple blaue Flecken & $48 \%$ \\
\hline Stuhlgang- und Verdauungsprobleme & $42 \%$ \\
\hline Gynäkomastie & $39 \%$ \\
\hline Dupuytrensche Kontrakturen & $23 \%$ \\
\hline Persistierende Diarrhoe & $22 \%$ \\
\hline Rezidivierendes Nasenbluten & $19 \%$ \\
\hline Anhaltende Kopfschmerzen & $7 \%$ \\
\hline Genitalmykose & $5 \%$ \\
\hline Rezidivierende Herzbeschwerden & $5 \%$ \\
\hline Rezidivierende Blasenentzündungen & $4 \%$ \\
\hline Karpaltunnelsyndrom & $4 \%$ \\
\hline
\end{tabular}

\section{Hausärztliches Zuweisungsverhalten}

Nach der Feststellung moderat erhöhter Leberwerte ziehen es 59 \% der befragten Hausärzte vor, zunächst ein abwartendes Offenhalten zu praktizieren und damit erst nach einer erneuten Untersuchung zu einem späteren Zeitpunkt eine Überweisung an eine höhere Fachgebietsebene zu erwägen. Etwa ein Viertel (27\%) hält eine unmittelbare Überweisung ohne Zuwarten und Wiederholung des Labors für angeraten (14\% unentschieden). Während 80 \% der Ärzte in Landgemeinden (bis 5000 Einwohner) ein kontrolliertes Zuwarten grundsätzlich vorziehen, beträgt der Anteil unter Ärzten in Großstädten $50 \%$.

Ein adäquater Zeitraum für das kontrollierte Zuwarten liegt nach Einschätzung der Befragten bei durchschnittlich 5,7 Wochen (Median: 5,0; Minimum: 1, Maximum: 19). Ärzte aus ländlichkleinstädtischen Umgebungen bevorzugen im Durchschnitt längere Wartedauern (7,2 Wochen) als Ärzte aus mittel- und großstädtischen Praxisumgebungen (4,2 Wochen).

Präferieren die meisten Hausärzte im Fall moderat erhöhter Leberwerte zunächst prinzipiell ein kontrolliertes Zuwarten (59\%), machen die Befragungsteilnehmer abweichende Angaben über ihr tatsächliches Überweisungsverhalten. Diesbezüglich geben $36 \%$ an, die Patienten in der Regel nach Feststellung unklar erhöhter Leberwerte direkt zum Facharzt bzw. an eine Spezialam- 
- Tab. 2 Betrachtete Laborwerte. Frage: Welche Laborwerte, die im Zusammenhang mit Lebererkrankungen stehen können, untersuchen Sie üblicherweise bei einer allgemeinen Screeninguntersuchung im Rahmen des „Routine-Labors“ bei Ihren Patienten? ( $\mathrm{N}=2.701)$.

\begin{tabular}{|c|c|c|c|c|}
\hline & \multirow[b]{2}{*}{ Gesamtzustimmung } & \multicolumn{3}{|c|}{ Rotierte Komponentenmatrix } \\
\hline & & $\begin{array}{l}\text { Komp. } 1 \\
\text { (Varianzaufkl.: 26,1 \%) }\end{array}$ & $\begin{array}{l}\text { Komp. } 2 \\
\text { (Varianzaufkl.: 18,2\%) }\end{array}$ & $\begin{array}{l}\text { Komp. } 3 \\
\text { (Varianzaufkl.: 10,6\%) }\end{array}$ \\
\hline $\begin{array}{l}\text { Alanin-Aminotransferase (ALAT, ALT, } \\
\text { GPT) }\end{array}$ & $63 \%$ &,- 055 & ,774 &,- 119 \\
\hline Y-Glutamyl-Transferase (GGT) & $95 \%$ &,- 040 &,- 018 & 884 \\
\hline $\begin{array}{l}\text { Aspartat-Aminotransferase (ASAT, } \\
\text { AST, GOT) }\end{array}$ & $65 \%$ & 141 &, 576 & ,386 \\
\hline AP (alkalische Phosphatase) & $62 \%$ &, 542 & 252 & ,305 \\
\hline Ferritin & $26 \%$ & ,734 &, 035 &,- 102 \\
\hline Bilirubin & $46 \%$ & ,686 & 219 & 213 \\
\hline Quick (INR) & $27 \%$ & ,663 & ,207 &,- 165 \\
\hline Cholinesterase & $19 \%$ & ,675 & ,139 &,- 023 \\
\hline Albumin & $23 \%$ & ,740 &,- 027 & 092 \\
\hline Thrombozyten & $57 \%$ & 256 & ,715 &,- 112 \\
\hline \multirow[t]{2}{*}{$\mathrm{MCV}$} & $55 \%$ & , 192 & ,614 & , 165 \\
\hline & & \multicolumn{3}{|c|}{$\begin{array}{l}\text { Extraktionsmeth.: Hauptkomponentenanalyse } \\
\text { Rotationsmeth.: Varimax, Kaiser-Normalisierung } \\
\text { Rotation in } 4 \text { Iterationen konvergiert } \\
\text { Aufgeklärte Gesamtvarianz: 54,9\% } \\
\text { Stichprobeneignung nach Kaiser-Meyer-Olkin: ,787 } \\
\text { Signifikanz nach Bartlett: p<0,001 }\end{array}$} \\
\hline
\end{tabular}

bulanz überwiesen zu haben. Nur $32 \%$ haben hingegen erst einmal konsequent zugewartet (30\% teils, teils; $2 \%$ keine Angabe).

$79 \%$ der Hausärzte geben an, die eigenen Patienten im Fall einer Überweisung bei einer gastroenterologischen Schwerpunktpraxis vorgestellt zu haben. $44 \%$ haben direkt an eine Leberspezialambulanz überwiesen (davon 55 \% der Ärzte in Groß- bzw. Mittelstädten und $35 \%$ der Ärzte in Landgemeinden bzw. Kleinstädten) sowie $27 \%$ zu einer gastroenterologischen Abteilung oder Klinik (Mehrfachnennungen waren möglich).

\section{Kooperation mit anderen Versorgungsebenen}

50 \% der befragten Hausärzte beurteilen die Zusammenarbeit mit niedergelassenen Gastroenterologen bei der Abklärung unklarer Leberwerterhöhungen oder der Diagnosestellung Leberzirrhose als sehr gut oder eher gut. Dagegen bewerten $43 \%$ die Kooperation als eher schlecht. Während $28 \%$ der Allgemeinmediziner in Großstädten die Kooperation negativ einschätzen, gibt demgegenüber jeder zweite Hausarzt (47\%) in Landgemeinden, Kleinund Mittelstädten ein negatives Urteil ab.

Im Prozess der ursprünglichen Fragebogenentwicklung wurde eine Fokusgruppe gebildet, in der mit Hausärzten themenbezogen diskutiert wurde. Aus der Diskussion sind verschiedene Problempunkte zu möglichen Schnittstellenproblematiken aufgegriffen und im Fragebogen zur Abstimmung gestellt worden. Die Ergebnisse zeigen, dass Hausärzte bei der Abklärung möglicher Leberkrankheiten verbreitete Hindernisse erleben. Diese betref- fen insbesondere fachärztliche Terminengpässe und die Verfügbarkeit gastroenterologischer Spezialisten, aber auch Herausforderungen in der interdisziplinären Kommunikation sowie der Arzt-Patient-Kommunikation (vgl. > Tab.3).

55 \% der in Großstädten niedergelassenen Allgemeinmediziner monieren, es gebe häufig oder gelegentlich zu wenige spezialisierte internistische Praxen in der Nähe, bei denen eine adäquate Abklärung von Leberwerten möglich sei, während in Landgemeinden $85 \%$ diese Sichtweise vertreten.

\section{Optimierungsansätze für die hausärztliche Versorgung}

Die Befragten erhielten eine Auflistung verschiedener möglicher Maßnahmen, um den Frühdiagnose-Anteil von Patienten zu erhöhen. Eingedenk des heterogenen Vorgehens bei der Untersuchung von Leberwerten und bestehender Schnittstellenproblematiken wird dabei die Etablierung eines strukturierten, evidenzbasierten Diagnose- und Therapiealgorithmus von $80 \%$ der Hausärzte als sehr effektive oder eher effektive Unterstützung beurteilt. $70 \%$ halten einen deutlichen Ausbau an Fortbildungsveranstaltungen für Hausärzte zur Abklärung von Leberwerten für sehr effektiv oder effektiv. $65 \%$ sehen eine Erweiterung der Laboruntersuchung im Rahmen der Gesundheitsuntersuchung ab dem 35. Lebensjahr als wirksame Maßnahme, während sich $61 \%$ die Entwicklung einer explizit hausarztkonformen, evidenzbasierten S3-Leitlinie zur systematischen Abklärung von Leber- 
- Tab. 3 Schnittstellenprobleme aus hausärztlicher Sicht. Frage: Bei der Zusammenarbeit zwischen Hausärzten und Fachärzten im ambulanten Bereich zur Abklärung einer Leberzirrhose können verschiedene Schwierigkeiten auftreten. Wie häufig treten die folgenden Schwierigkeiten Ihrer Erfahrung nach auf? $(\mathrm{N}=2.701)$.

\begin{tabular}{|c|c|c|c|c|c|}
\hline Aussage & Häufig & Gelegentlich & Selten & Nie & $\begin{array}{l}\text { Keine } \\
\text { Angabe }\end{array}$ \\
\hline $\begin{array}{l}\text { Die niedergelassenen gastroenterologischen Fachärzte sind aufgrund } \\
\text { der zahlreichen erforderlichen Gastroduodenoskopien und Kolosko- } \\
\text { pien auf lange Zeit ausgebucht. }\end{array}$ & $69 \%$ & $21 \%$ & $6 \%$ & $3 \%$ & $1 \%$ \\
\hline $\begin{array}{l}\text { Fachärzten fehlt die erforderliche Zeit, um sich mit ihnen über die } \\
\text { meist komplexen Patientenprobleme auszutauschen. }\end{array}$ & $41 \%$ & $39 \%$ & $10 \%$ & $8 \%$ & $2 \%$ \\
\hline $\begin{array}{l}\text { Es gibt zu wenige spezialisierte internistische Praxen in der Nähe, bei } \\
\text { denen eine Abklärung von Leberwerten in der von mir gewünschten } \\
\text { Weise möglich ist. }\end{array}$ & $37 \%$ & $36 \%$ & $11 \%$ & $15 \%$ & $1 \%$ \\
\hline $\begin{array}{l}\text { Die Patienten werden vom Facharzt nicht ausreichend über ihre Situa- } \\
\text { tion aufgeklärt und kehren aus Unsicherheit zum Hausarzt zurück. }\end{array}$ & $30 \%$ & $42 \%$ & $13 \%$ & $13 \%$ & $2 \%$ \\
\hline $\begin{array}{l}\text { Gastroenterologische Fachärzte sind für die Patienten schlecht } \\
\text { erreichbar. }\end{array}$ & $35 \%$ & $34 \%$ & $16 \%$ & $14 \%$ & $1 \%$ \\
\hline $\begin{array}{l}\text { Im Fall eines Verdachts auf Leberzirrhose stellt der Facharzt keine } \\
\text { direkte Überweisung zu einem Leberzentrum aus, sodass der Patient } \\
\text { vorerst zum Hausarzt zurückkehrt (Schleife und Zeitverlust). }\end{array}$ & $23 \%$ & $40 \%$ & $20 \%$ & $16 \%$ & $1 \%$ \\
\hline $\begin{array}{l}\text { Die Termine bei Fachärzten sind auf zu lange Zeit ausgebucht, sodass } \\
\text { ich den Patienten direkt in einer klinischen Spezialambulanz vorstelle. }\end{array}$ & $21 \%$ & $35 \%$ & $19 \%$ & $23 \%$ & $2 \%$ \\
\hline Man muss lange warten, bis die Fachärzte ihre Befunde weiterleiten. & $19 \%$ & $33 \%$ & $20 \%$ & $27 \%$ & $1 \%$ \\
\hline $\begin{array}{l}\text { Fachärzte informieren den Hausarzt nicht ausreichend über die von } \\
\text { ihnen durchgeführten Untersuchungen, Ergebnisse und/oder gestellten } \\
\text { Diagnosen. }\end{array}$ & $17 \%$ & $35 \%$ & $24 \%$ & $23 \%$ & $1 \%$ \\
\hline
\end{tabular}

werterhöhungen wünschen. 50 \% sprechen sich für die Einführung eines genuinen Leber-Checks im Rahmen der GKV aus.

Weitere Erhebungen weisen darauf hin, dass Hausärzte sich der Wichtigkeit des Themas Früherkennung von Leberkrankheiten bewusst sind und die eigenen Kenntnisse in diesem Anwendungsfeld insgesamt eher selbstkritisch einschätzen. So halten sich $38 \%$ bei der Abklärung von Leberwerterhöhungen für sehr oder eher kompetent, während $46 \%$ sich als weniger kompetent einstufen (16\% keine Angabe).

\section{Diskussion}

\section{Zusammenfassung und Befunde anderer Studien}

Die Ergebnisse der Befragung von 2.701 Hausärzten in zwei großen Flächenländern bestätigen, dass Leberwerterhöhungen ein häufiger Befund in der primärärztlichen Versorgung sind. Damit geht die Notwendigkeit einer systematischen und konsequenten Abklärung einher. Die Befragungsergebnisse untermauern in vielen Bereichen die Befunde der Vorstudie [30] und liefern Hinweise, dass beim Management erhöhter Leberwerte in der hausärztlichen Versorgung eine Reihe von Schwachpunkten besteht.

- Erstens zeigt sich ein heterogenes und divergierendes hausärztliches Vorgehen bei der Abklärung einer möglichen chronischen Leberparenychmerkrankung. So achten Allgemeinmediziner nicht nur auf stark unterschiedliche Symptome, sondern zie- hen im Rahmen der hausärztlich veranlassten Labordiagnostik unterschiedliche leberassoziierte Laborparameter bzw. Wertekonstellationen als Indikatoren zur Identifikation einer (beginnenden) Leberkrankheit heran. Während eine Gruppe von Hausärzten sich auf Funktionsparameter konzentriert, betrachtet eine andere in erster Linie Indikatoren für eine toxische Zellschädigung oder eine schon eingetretene Lebererkrankung [30]. Die drei bereits in der Vorstudie identifizierten Cluster bei der Laborwertanalyse konnten nun erneut nachgewiesen werden. Diese dürften damit zusammenhängen, dass die beauftragten Labore keine deckungsgleichen Portfolios zur Verfügung stellen. Zugleich scheint es bei einem Teil der Hausärzte die Bestrebung zu geben, im Praxisalltag auf möglichst wenige griffige Parameter zu fokussieren. Insbesondere die $y$-GT scheint dabei für viele Hausärzte ein naheliegender und oft singulär betrachteter Indikator zu sein, obwohl eine isolierte Erhöhung der $\mathrm{y}$-GT ohne Alkoholkonsum nicht zwangsläufig auf eine Leberpathologie hindeutet [31]. Auch eine Befragung niedergelassener Gastroenterologen hat bestätigt, dass sich Allgemeinmediziner im Praxisalltag an stark unterschiedlichen Leberwerten orientieren [32]. Entsprechend divers stellt sich auch das Spektrum für Schlussfolgerungen und weitere Versorgungsentscheidungen dar.

- Eine zweite Problematik betrifft die Frage, inwiefern nach Feststellung erhöhter Leberwerte kontrolliert zugewartet wird und ab welchem Zeitpunkt eine Überweisung angezeigt ist. Zwar bevorzugt grundsätzlich eine Mehrzahl der Befragten ein kon- 
trolliertes Zuwarten von bis zu acht Wochen, allerdings liefert die Befragung Hinweise, dass Hausärzte im Versorgungsalltag von rascheren und häufigeren Überweisungen zu Fachärzten oder Leberambulanzen Gebrauch machen. Dabei praktizieren Ärzte mit ländlichem Praxisstandort ein längeres Zuwarten als Ärzte in Großstädten. Wie andere Arbeiten zeigen, erfolgen der Einsatz von diagnostischen Verfahren und die Vorstellung von Patienten bei Fachärzten im Fall von Landärzten zurückhaltender als bei Medizinern in städtischen Gebieten [33].

- Drittens gibt es Anhaltspunkte dafür, dass Hausärzte beim betrachteten Thema Unsicherheit und einen Mangel an Orientierung verspüren. Dafür spricht etwa die Selbsteinschätzung der Ärzte, aber auch die Tatsache, dass Praxisempfehlungen und Expertisen medizinischer Fachgesellschaften eher selten genutzt werden. Das artikulierte Interesse an einem Ausbau adäquater Fortbildungsangebote ist ebenfalls ein Indiz für einen themenbezogenen Schulungsbedarf.

- Viertens bestehen aus hausärztlicher Perspektive Herausforderungen in der interdisziplinären Zusammenarbeit zwischen Hausärzten und niedergelassenen internistisch-gastroenterologischen Fachärzten. Vor allem die mangelnde Erreichbarkeit und der Umstand, dass es relativ häufig zu längeren Wartezeiten auf einen Termin zur differenzialdiagnostischen Klärung beim Verdacht auf eine Leberkrankheit kommt, stellen Erschwernisse für Hausärzte dar. Im ländlichen und kleinstädtischen Bereich stellen sich einige Herausforderungen in Anbetracht der deutlich niedrigeren Facharztdichte in verschärfter Weise dar [34]. Indes zeigt eine Befragung von gastroenterologischen Fachärzten in Rheinland-Pfalz, dass diese ihrerseits Kritik am hausärztlichen Zuweisungsverhalten üben, das nach Auffassung dieser Befragten häufig entweder deutlich verfrüht oder zu stark verzögert ist [32]. Somit könnten möglicherweise eine oft unreflektierte Überweisung durch Hausärzte und der Verzicht auf eine Basisabklärung der Ursachen ein bedeutendes Problem in der Interaktion mit Fachärzten sein.

Der Umgang mit erhöhten Leberwerten, die im Rahmen einer allgemeinen Blutuntersuchung auffallen, stellt eine diagnostische Herausforderung dar, die bislang stark vom individuellen Vorgehen des einzelnen Hausarztes abhängt. Die Ergebnisse können mit dem Umstand in Zusammenhang gestellt werden, dass bislang ein validierter, breit akzeptierter diagnostischer Algorithmus für die Identifizierung von Patienten mit erhöhten Leberwerten bei gleichzeitig hohem Risiko für eine Leberzirrhose für die hausärztliche Versorgung fehlt [25-28]. Ein solcher in der Breite der Versorgung angewandter strukturierter Diagnose- und Behandlungspfad könnte ein wertvolles Tool zu Professionalisierung und Vereinheitlichung des hausärztlichen Vorgehens sein [22, 35].

Es liegen bereits Vorschläge vor, wie ein systematisches und praktikables diagnostisches Vorgehen für einen solchen Behandlungspfad ablaufen könnte [36-39]. Holstege kategorisiert etwa Vorgehensweisen anhand des Musters der pathologisch veränderten Leberwerte in drei verschiedene Gruppen [40]. Bei Erhöhung der Transaminasen sollte zunächst geklärt werden, ob eine virale Genese, eine genetisch bedingte Stoffwechselerkrankung oder eine medikamentös-toxische Schädigung vorliegt. Im Fall einer Erhöhung von Cholestaseenzymen sollte mittels Sonografie un- tersucht werden, ob eine intra- oder extrahepatische Ursache der Cholestase vorliegt. Bei einem Anstieg der $\mathrm{\gamma}$-GT ist verstärkt auf extrahepatische Krankheitsursachen und physiologische Enzymerhöhungen zu achten. Die Entwicklung und breite Etablierung eines praxisnahen, situationsbezogenen Algorithmus zur (weiteren) Abklärung erhöhter Leberwerte wäre nicht zuletzt ein wertvoller Beitrag, um Schnittstellenproblematiken entgegenzuwirken [17]. So ließe sich das differenzialdiagnostische Vorgehen besser strukturieren, der Informationsfluss optimieren und eine reibungslosere Arbeitsteilung zwischen Haus- und Fachärzten sicherstellen [32].

In ähnlicher Größenordnung wie in der Vorstudie sprechen sich in der Befragung vier von fünf Hausärzten für die Einführung eines strukturierten Diagnosealgorithmus aus und erachten diesen nicht als Eingriff in die ärztliche Therapiefreiheit [30, 32]. Eine wichtige Voraussetzung für die erfolgreiche Durchsetzung eines solchen Instruments wird indes sein müssen, dass es in der Lage ist, sich möglichst dicht an der hausärztlichen Versorgungsrealität zu orientieren.

\section{Stärken und Schwächen}

Die Befragung war aufgrund mehrerer Vorstudien bereits konzeptionell erprobt und auf das allgemeinärztliche Versorgungsgeschehen zugeschnitten. Im Zuge der Durchführung konnte zudem eine große, heterogene und breit gestreute Stichprobe aus zwei Bundesländern gewonnen werden, die ein breites Bild des hausärztlichen Umgangs und entsprechender Voraussetzungen bei der Leberwertabklärung eröffnet. Dennoch kann die Studie keinen repräsentativen Anspruch erheben, da sie einen regionalen Schwerpunkt und einen begrenzten Rücklauf hatte. Weiter ist es aufgrund der Anonymisierung - die als Voraussetzung für eine breite Teilnahme diente - nicht möglich, zurückzuverfolgen, aus welchen Teilen der beiden Bundesländer Hausärzte teilgenommen haben. Auch kann nicht ausgeschlossen werden, dass sich thematisch interessierte Ärzte in stärkerem Maße zur Teilnahme bereiterklärt haben.

Mit Blick auf die Limitationen der Studie ist anzumerken, dass sie sowohl anamnestisch als auch von der Befundseite die alkoholische Lebererkrankung stärker betont und demgegenüber das hepatologische Problem der Fettleber, virale Lebererkrankungen und systemische Autoimmunphänomene weniger Beachtung finden. Somit kann die Befragung der Breite von Lebererkrankungen in der hausärztlichen Versorgung nicht umfänglich gerecht werden. Diese Forschungslücke zu bearbeiten wird Aufgabe von Folgearbeiten sein müssen.

Bei mehreren der Fragemodelle wurden Anamnese und Befunde nicht immer klar getrennt, was bei der Interpretation der Ergebnisse berücksichtigt werden muss. Zudem wären komplexere Abfragen denkbar gewesen, die ein differenzierteres Bild des diagnostischen Vorgehens ermöglicht hätten (z. B. Verhalten beim kontrollierten Zuwarten). Auch ist es möglich, dass bei bestimmten Fragen (z. B. zur interdisziplinären Zusammenarbeit) durch negativ formulierte Items bei manchen Befragten ein suggestiver Effekt bezüglich der problemaggravierenden Antworten ausgelöst wurde. 
Für künftige Studien wäre interessant zu erfassen, welche Maßnahmen aus Sicht von Hausärzten mit hepatologischer Kompetenz dazu geführt haben, sicherer im Umgang mit Leberwerterhöhungen zu werden, und welche Maßnahmen darüber hinaus aus Sicht dieser Behandler ergriffen werden sollten, um die Effektivität der hausärztlichen Früherkennung zu optimieren.

\section{Schlussfolgerungen}

Erhöhte Leberwerte treten als häufiger Zufallsbefund in der hausärztlichen Versorgung auf. Umso größer ist die Bedeutung, die einer effektiven Abklärungs- und Ausschlussdiagnostik zukommt, um zu verhindern, dass vorhandene Leberkrankheiten übersehen werden. Dabei ist nicht nur relevant, welche Leberwerte in welchen Konstellationen herangezogen oder wann Patienten zur weiterführenden Untersuchung überwiesen werden. Ebenso ist die Qualität einer funktionierenden Kooperation zwischen Haus- und Facharztebene entscheidend.

Die Studienergebnisse weisen darauf hin, dass die frühzeitige, konsequente Identifizierung und Abklärung (unklarer) Leberwerterhöhungen in der Hausarztpraxis derzeit nicht immer möglich ist, da verschiedene Hürden und Herausforderungen bestehen. Um die Effektivität der primärärztlichen Versorgung sukzessive zu steigern, erscheint es ratsam, Maßnahmen zu ergreifen, die zu einer verstärkten Professionalisierung und Vereinheitlichung der Diagnostik beitragen und die Zusammenarbeit mit gastroenterologischen Spezialisten besser strukturieren. Vorstellbar wäre dazu ein breiteres Angebot von Schulungs- und Fortbildungsformaten, die Entwicklung eines validierten Diagnose- und Behandlungspfads oder die feste Verankerung einer leberwertassoziierten Blutuntersuchung im Rahmen des Check-ups. Die Entwicklung einer fundierten hausarztbasierten Leitlinie zur Erkennung von und zum Umgang mit erhöhten Leberwerten erscheint ratsam. Auch ist in Anbetracht der stark gestiegenen Zahl der von Adipositas und Diabetes betroffenen Patienten eine sinnvolle Selektion der Patienten mit einem weiteren fachärztlichen Abklärungsbedarf aus Kapazitätsgründen relevant.

\section{Einhaltung ethischer Richtlinien}

Bei der vorliegenden Studie wurden keinerlei sensible Patientendaten erhoben oder klinische Tests durchgeführt. Dennoch haben sich die Autoren im Vorfeld der Studie mündlich bei der Ethikkommission des Bundeslandes Rheinland-Pfalz rückversichert, dass die Studie der Berufsordnung für Ärzte gerecht wird.

Dieser Beitrag umfasst keine Studien an Menschen oder Tieren.

Interessenkonflikt

Die Autorinnen/Autoren geben an, dass kein Interessenkonflikt besteht.

\section{Literatur}

[1] Lobstein S, Kaiser T, Liebert U et al. Prevalence, aetiology and associated co-morbidities of elevated aminotransferases in a german cohort of orthopaedic surgery patients. Z Gastroenterol 2008; 46: 415-420. doi:10.1055/s-2008-1027157
[2] Schattenberg J, Wild P, Zeller T et al. Prävalenz erhöhter GPT- und gGTWerte und assoziierte Risikofaktoren - eine Querschnittsanalyse von 5000 Teilnehmern der Gutenberg Herz Studie. Z Gastroenterol 2011; 49: P5_30 doi:10.1055/s-0030-1269736

[3] Baumeister SE, Volzke $\mathrm{H}$ et al. Impact of fatty liver disease on health care utilization and costs in a general population: a 5-year observation. Gastroenterology 2008; 134: 85-94. doi:10.1053/j.gastro.2007.10.024

[4] Keitel V, vom Dahl S, Häussinger D. Sekundäre Ursachen der Fettleber Neues zur Pathogenese, rationalen Diagnostik und Therapie. Dtsch Med Wochenschr 2020; 145: 140-145. doi:10.1055/a-0965-9648

[5] Ruhl CE, Everhart JE. Elevated serum alanine aminotransferase and gamma-glutamyltransferase and mortality in the United States population. Gastroenterology 2009; 136: 477-85.e11. doi:10.1053/ j.gastro.2008.10.052

[6] Tae HL, Kim WR, Benson JT et al. Serum aminotransferase activity and tality risk in a United States community. Hepatology 2008; 47: 880-887. doi:10.1002/hep.22090

[7] Fulks M, Stout R, Dolan V. Using liver enzymes as screening tests to predict mortality risk. Journal of insurance medicine 2008; 3-4: 191-203

[8] Koehler E, Sanna D, Hansen B et al. Serum liver enzymes are associated with all-cause mortality in an elderly population. Liver international 2014; 2: 296-304

[9] Kunutsor S, Apekey T, Seddoh D et al. Liver enzymes and risk of all-cause mortality in general populations. Int J Epidemiol 2014; 1: 187-201

[10] Pratt D, Kaplan M. Evaluation of abnormal liver-enzyme results in asymptomatic patients. N Engl J Med 2000; 342: 1266-1271. doi:10.1056/NEJM200004273421707

[11] Green R, Flamm S. AGA technical review on the evaluation of liver chemistry tests. Gastroenterology 2002; 4: 1367-1384. doi:10.1053/ gast.2002.36061

[12] Chadwick A, Marks M. Low yield of unselected testing in patients with acutely abnormal liver function tests. JRSM open 2016; 1 : 2054270415611309 doi: $10.1177 / 2054270415611309$

[13] Lawrence S. Friedman. Approach to the patient with abnormal liver biochemical and funstion tests. Im Internet (Stand: 01.07.2020): https://www.uptodate.com/contents/approach-to-the-patient-withabnormal-liver-biochemical-and-function-tests

[14] Bahr M. Abklärung erhöhter Leberwerte. Dtsch Med Wochenschr 2013; 138: 131-148. doi:10.1055/s-0032-1327255

[15] Neumaier J. Erhöhte Leberwerte. Der Hausarzt 2017; 8: 61-62

[16] Zimmermann HW, Tacke F, Kroy D et al. Gastroenterologie: Erhöhte Leberwerte - was nun? Dtsch Arztebl 2016; 113: A-1104

[17] Ebner H, Schuchmann M. Erhöhte Leberwerte. Wann harmlos, wann kritisch? Der Allgemeinarzt 2019; 41: 24-28

[18] Töx U. Rationelle Diagnostik bei erhöhten Leberwerten. Med Welt 2009; 60: $121-127$

[19] Bettinger D, Thimme R. Praktisches Vorgehen bei Erstdiagnose einer Leberzirrhose. Dtsch Med Wochenschr 2019; 144: 1251-1258. doi:10.1055/a-0753-6021

[20] Jepsen P, Ott P, Andersen PK et al. Clinical course of alcoholic liver cirrhosis: a Danish population-based cohort study. Hepatology 2010; 51: 1675-1682. doi:10.1002/hep.23500

[21] Blachier M, Leleu H, Peck-Radosavljevic M et al. The burden of liver disease in Europe: a review of available epidemiological data. J Hepatol 2013; 58: 593-608. doi:10.1016/j.jhep.2012.12.005

[22] Wedemeyer $\mathrm{H}$, Hofmann WP, Lueth $\mathrm{S}$ et al. ALT screening for chronic liver diseases: Scrutinizing the evidence. Z Gastroenterol 2010; 48: 4655. doi:10.1055/s-0028-1109980

[23] Wiegand J, Berg T. The etiology, diagnosis and prevention of liver cirrhosis: part 1 of a series on liver cirrhosis. Dtsch Arzteblatt Int 2013; 110 : 85-91. doi:10.3238/arztebl.2013.0085 
[24] Dufour JF, Greten TF, Raymond E et al. Clinical Practice Guidelines EASL EORTC Clinical Practice Guidelines: Management of hepatocellular carcinoma. European Organisation for Research and Treatment of Cancer. J Hepatol 2012; 56: 908-943. doi:10.1016/j.jhep.2011.12.001

[25] Chang T, Liaw Y, Wu S et al. Long-Term Entecavir Therapy Results in the Reversal of Fibrosis/Cirrhosis and Continued Histological Improvement in Patients with Chronic Hepatitis B. Hepatology 2010; 52: 886-893. doi: $10.1002 /$ hep. 23785

[26] Zoutendijk R, Reijnders JGP, Zoulim F et al. Virological response to entecavir is associated $\mathrm{h}$ a better clinical outcome in chronic hepatitis $\mathrm{B}$ patients with cirrhosis. Gut 2013; $62: 760$ doi:10.1136/gutjnl-2012302024

[27] Garcia-Tsao G, Sanyal A], Grace ND et al. Prevention and management of gastroesophageal varices and variceal hemorrhage in cirrhosis. Hepatology 2007; 46: 922-938. doi:10.1002/hep.21907

[28] Colombo M. Screening and diagnosis of hepatocellular carcinoma. Liver Int 2009; 29 (212): (Suppl. 1): 143-147. doi:10.1111/ j.1478-3231.2008.01938.x

[29] Fromm S. Faktorenanalyse. In: Baur N, Fromm S, Hrsg. Datenanalyse mit SPSS für Fortgeschrittene. Ein Arbeitsbuch. Wiesbaden: Springer; 2008: 314-344

[30] Jansky M, Mattlinger C, Nguyen-Tat M et al. Abklärung von Leberwerterhöhungen in der hausärztlichen Praxis - Versorgungsrealität in Deutschland. Deut Med Wochenschr 2018; 143: 34-41. doi:10.1055/ s-0043-122251

[31] Ärzte Zeitung. Gamma-GT. Schlechtes Zeichen, aber nicht für die Leber. Im Internet (Stand: 01.07.2020): https://www.aerztezeitung.de/ Medizin/Schlechtes-Zeichen-aber-nicht-fuer-die-Leber-265985.html
[32] Jansky M, Wangler ], Nguyen-Tat M et al. Abklärung erhöhter Leberwerte - Wie erleben gastroenterologische Fachärzte die Zusammenarbeit mit Hausärzten? Z Allg Med 2018; 94: 494-499. doi:10.3238/ zfa.2018.0494-0499

[33] Spoont M, Greer N, Su J et al. Rural vs. Urban Ambulatory Health Care: A Systematic Review (2011). Im Internet (Stand: 18.05.2020): https://www.ncbi.nlm.nih.gov/pubmed/21735564

[34] Gesundheitsberichterstattung des Bundes. Angebot ambulanter ärztlicher Versorgung - Entwicklung im städtischen und ländlichen Raum. Im Internet (Stand: 01.07.2020): www.gbe-bund.de

[35] Furger P, Schaufelberger M. Algorithmen quick für den Hausarzt. Flussdiagramme zu Differentialdiagnose und Befundabklärung. Stuttgart: Thieme; 2011

[36] American Gastroenterological Association. Medical position statement: evaluation of liver chemistry tests. Gastroenterology 2002; 123: 13641366. doi:10.1053/gast.2002.36060

[37] Giannini EG, Testa R, Savarino V. Liver enzyme alteration: a guide for clinicians. CMAJ 2005; 172: 367-379. doi:10.1503/cmaj.1040752

[38] Green RM, Flamm S. AGA technical review on the evaluation of liver chemistry tests. Gastroenterology 2002; 123: 1367-1384. doi:10.1053/ gast.2002.36061

[39] Clark JM, Brancati FL, Diehl AM. The prevalence and etiology of elevated aminotransferase levels in the United States. Am J Gastroenterol 2003; 98: 960-967. doi:10.1111/j.1572-0241.2003.07486.x

[40] Holstege A. Erhöhte Leberwerte. Dtsch med Wochenschr 2016; 141 : 1640-1646. doi:10.1055/s-0042-100041 